\title{
GESTIÓN LOCAL DEL TURISMO: EL MUNICIPIO Y SU ROL EN EL DESARROLLO TURÍSTICO EN CHILE.
}

\author{
Javier Rodríguez Valenzuela \\ Investigador del Centro de Investigación en Turismo y Patrimonio (CITYP) \\ Socioeconomista con Mención en Desarrollo Local \\ Magister en Análisis Sistémico Aplicado a la Sociedad \\ Javier. Rodríguez@cityp.org
}

\section{RESUMEN}

El presente trabajo es una investigación de carácter descriptivo, el cual tiene como objetivo analizar el rol de los municipios en el desarrollo turístico en Chile por medio de la identificación de las atribuciones, funciones y caracterización de la gestión que a nivel local están desarrollando. El proceso de producción de información se realizó a través de una estrategia metodológica cualitativa, utilizando como técnicas de producción de información la revisión documental y la etnografía. Entre los principales resultados se encuentra la identificación de nueve áreas de acción. Con relación a las atribuciones que poseen los municipios, resalta su característica como actividad "no exclusiva", apreciando la complejidad a partir de la multiplicidad de actores que intervienen en los procesos de desarrollo turístico a nivel local. Se identificó la carencia existente a nivel nacional de instrumentos de planificación turística, observando la necesidad de que se reconozcan por la actual legislación de municipalidad, ya que contar con un (PLADETUR) facilitaría la adopción de las exigencias actuales de la industria, vinculadas a desarrollar la actividad bajo un modelo de sustentabilidad. Finalmente y con relación a la caracterización de la gestión local, se visualiza la necesidad de incrementar los recursos humanos y financieros que disponen los municipios en la actualidad.

Palabras Claves: Municipalidad - Turismo - PLADETUR - Desarrollo Local Sustentabilidad 


\title{
TOURISM LOCAL MANAGEMENT: MUNICIPALITIES AND THEIR ROLE ON THE TOURISTIC DEVELOPMENT IN CHILE.
}

\author{
Javier Rodríguez Valenzuela \\ Investigador del Centro de Investigación en Turismo y Patrimonio (CITYP) \\ Socioeconomista con Mención en Desarrollo Local \\ Magister en Análisis Sistémico Aplicado a la Sociedad \\ Javier. Rodríguez@cityp.org
}

\begin{abstract}
This article aims to analyze the role of municipalities on tourism development in Chile with emphasis on the identification of attributions, functions and characteristics of local tourism management. A qualitative approach is adopted to collect and analyze data based on both, the techquique of ethnography and documentary review. Among the main results, nine areas of action are identified for local tourism management. In relation with the attributions that the municipalities have, the results show that tourism is a "nonexclusive" activity for the municipality. This might be explained because of the complexity resulting from the wide range of actors intervening on a tourism planning process at the local level. The lack of tourism planning instruments at the national level was also identified. In fact, the existing planning tools, such as PLADETUR, are not recognized at the municipal legislation. This is critical because PLADETUR would facilitate the adoption of requirements of the industry and promote sustainability tourism development. Finally, regarding tourism local management, the results show the need to increase human and financial resources in municipalities in order to tackle tourism development.
\end{abstract}

Key Words: Municipality - Tourism - PLADETUR - Local development - Sustainability 


\section{INTRODUCCIÓN.}

En innegable apreciar la relevancia que posee el turismo en la actualidad para el crecimiento y desarrollo socioeconómico de diversos países a nivel global. Un dato que reafirma esta mirada, se vincula con el aumento de las llegadas de turistas internacionales en el mundo, las cuales crecieron un 4,4\% en el año 2015, alcanzando un total de 1.184 millones, significando un incremento de 50 millones más que en el año 2014 (OMT, 2016). Esta situacion genera un dinamismo económico y socio-cultural que son absorbidos por las comunidades y territorios locales a través del aumento sostenido de los flujos de demanda.

Esta situacion se puede observar en el caso de Chile específicamente, donde se visualiza un incremento en las llegadas de turistas internacionales durante los últimos cinco años. Esta situación se puede apreciar con lo acontecido durante el año 2015, el cual consolida un leve incremento observado en el año 2014, alcanzando un total de 4.478 .336 turistas, cifra que representa un $21,9 \%$ de incremento en comparación al año 2014. Este incremento posiciona la actividad turística como un sector clave para la economía interna, aportando en forma directa un 3,2\% al Producto Interno Bruto (PIB) y en un 10,2\% en forma total (WTTC,2016).

Si observamos la realidad Chilena y específicamente el planteamiento de la actual Ley de Turismo promulgada durante el año 2010, la cual indica que; "El turismo constituye una actividad estratégica para el desarrollo del país, siendo prioritaria dentro de las políticas de Estado, por lo que éste deberá promoverla de modo armónico e integral, impulsando su crecimiento sustentable en conformidad con las características de las regiones, comunas y localidades del país" (Ley de Turismo, 2010, Art.2).

Junto a esta política nacional de turismo, el Estado chileno ha venido elaborando e implementando diversas acciones para desarrollar la actividad turística. Entre las más relevantes acciones de la última década podemos mencionar la elaboración de la estrategia nacional de turismo 2012-2020 y el Plan Nacional de Desarrollo Turístico Sustentable.

Considerando estos antecedentes podemos deducir que el movimiento de turistas internacionales, el cual se compone por el turismo emisivo y receptivo (OMT,1997), más el turismo interno, seguirá incrementándose en el futuro, donde el papel que deben de cumplir los municipios como un actor clave para el desarrollo en el ámbito local debe potenciarse, ya que la diversidad geográfica de Chile continental, representada en los $4.300 \mathrm{~km}$ aproximadamente de longitud de norte a sur, más los $3.800 \mathrm{Km}$, si se incluye la zona antártica (Fundación Mar, 2005), lo hace poseedor de una riqueza patrimonial ligada a los paisajes y tradiciones, encontrando una variedad de destinos y productos turísticos "potenciales y consolidados" en las diversas comunas del pais, en los cuales se pueden vivenciar actividades vinculadas al turismo arqueológico, 
astronómico, de sol y playa, religioso, deportivo, enoturismo, ecoturismo, entre otros.

Si se observan los datos expuestos en el cuadro $\mathrm{N}^{\circ} 1$, se aprecia el dinamismo vinculado a la llegada de extranjeros a destinos vinculados al turismo tradicional $\mathrm{y}$ al de intereses especiales, mostrando este último un incremento en el turismo receptivo, focalizado en la zona sur y en la zona central.

\section{Cuadro $\mathrm{N}^{\circ}$ 1: Evolución de las llegadas de turistas extranjeros a destinos turísticos 2012 -2014, según datos de ingreso a chile por aeropuerto internacional.}

\begin{tabular}{|c|c|c|c|c|c|c|}
\hline Tipología Lugar Visitado & Lugar visitado & 2012 & 2013 & 2014 & $\begin{array}{c}\text { Variación porcentual } \\
2014 / 2012\end{array}$ & $\%$ \\
\hline $\begin{array}{l}\text { Destinos vinculados } \\
\text { al turismo tradicional }\end{array}$ & $\begin{array}{l}\text { Arica } \\
\text { Iquique - Salitreras - Pica - Mantilla } \\
\text { Antofagasta - Calama } \\
\text { La Serena - Coquimbo } \\
\text { Otros sectores costeros R. de Valpo. } \\
\text { Pichilemu - Constitucion } \\
\text { Valparaiso - Viña del Mar }\end{array}$ & $\begin{array}{r}23.386 \\
33.323 \\
52.115 \\
67.687 \\
100.620 \\
27.049 \\
549.502\end{array}$ & $\begin{array}{r}18.648 \\
28.999 \\
49.945 \\
73.461 \\
97.258 \\
21.137 \\
604.795\end{array}$ & $\begin{array}{r}20.167 \\
28.536 \\
49.056 \\
64.291 \\
122.765 \\
21.479 \\
634.274\end{array}$ & $\begin{array}{l}-13,8 \% \\
-14,4 \% \\
-5,9 \% \\
-5,0 \% \\
22,0 \% \\
-20,6 \% \\
15,4 \% \\
\end{array}$ & $\begin{array}{r}1,3 \% \\
1,9 \% \\
3,2 \% \\
4,2 \% \\
8,0 \% \\
1,4 \% \\
41,3 \% \\
\end{array}$ \\
\hline $\begin{array}{l}\text { Destinos vinculados } \\
\text { a intereses especiales }\end{array}$ & $\begin{array}{l}\text { San Pedro de Atacama - Géys eres del Tatio } \\
\text { Putre - Lago Chungará } \\
\text { Valle del Elqui - Vicuña } \\
\text { Isla de Pascua } \\
\text { Pucon - Villarrica - Lican Ray - Caburga } \\
\text { Valdivia - sector costero } \\
\text { Osorno y alrededores } \\
\text { Pto. Montt - Pto. Varas - P.N. Perez Rosales } \\
\text { Isla de Chiloé } \\
\text { Carretera Austral - Futaleufu - Palena } \\
\text { Torres del Paine } \\
\text { Punta Arenas } \\
\text { Centros Invernales }\end{array}$ & $\begin{array}{r}98.008 \\
6.555 \\
26.865 \\
30.089 \\
74.609 \\
42.296 \\
23.708 \\
102.672 \\
30.781 \\
12.588 \\
57.555 \\
56.391 \\
161.317\end{array}$ & $\begin{array}{r}96.667 \\
4.815 \\
24.532 \\
27.264 \\
77.411 \\
36.954 \\
22.608 \\
95.677 \\
29.930 \\
12.244 \\
59.126 \\
63.293 \\
146.602\end{array}$ & $\begin{array}{r}111.200 \\
6.626 \\
29.107 \\
34.921 \\
71.929 \\
36.098 \\
24.028 \\
113.452 \\
42.013 \\
15.422 \\
69.451 \\
83.361 \\
198.171\end{array}$ & $\begin{array}{c}13,5 \% \\
1,1 \% \\
8,3 \% \\
16,1 \% \\
-3,6 \% \\
-14,7 \% \\
1,4 \% \\
10,5 \% \\
36,5 \% \\
22,5 \% \\
20,7 \% \\
47,8 \% \\
22,8 \%\end{array}$ & $\begin{array}{r}7,2 \% \\
, 4 \% \\
1,9 \% \\
2,3 \% \\
4,7 \% \\
2,4 \% \\
1,6 \% \\
7,4 \% \\
2,7 \% \\
1,0 \% \\
4,5 \% \\
5,4 \% \\
12,9 \%\end{array}$ \\
\hline
\end{tabular}

Fuente: Sernatur.

Ahora bien, para que el incremento experimentado tenga un buen funcionamiento, se requiere inevitablemente poder generar procesos de desarrollo y planificación local, apreciando la relevancia que cumplen los municipios como actor clave en la gestión local del turismo y en la generación de procesos de adaptación a las nuevas exigencias de la industria, como lo es por ejemplo, poder desarrollar esta actividad con la participación activa de comunidades locales y bajo un modelo de turismo sustentable.

Considerando estos antecedentes, resulta de interés poder observar la rol de los municipios, asumiendo que en la sociedad compleja actual, son un importante agente de desarrollo a nivel local y portadores de herramientas y atribuciones para desarrollar e intervenir la actividad turística en los territorios. 


\subsection{Objetivos del Estudio.}

- Objetivo General: Analizar el rol que tienen los municipios en la gestión local del turismo en Chile.

\section{- Objetivos específicos:}

a) Identificar las funciones y atribuciones que poseen en la actualidad los municipios para desarrollar la actividad turística en Chile.

b) Identificar los instrumentos de planificación que poseen los municipios y su vinculación con la actividad turística.

c) Caracterizar la gestión turística de los municipios en Chile.

\section{METODOLOGÍA.}

El plan de acceso al problema de investigación se genera a partir de un diseño de tipo cualitativo, enmarcado en un tipo de estudio descriptivo, que contempla fuentes primarias y secundarias, utilizando como técnica la revisión documental y la etnografía (Valles, 1999).

El análisis documental se realizó a partir de un proceso de revisión de informes elaborados por el Servicio Nacional de Turismo (en versión digital) y de bibliografía atingente a la problemática a indagar, mediante un diseño de análisis de la información que se dividió en dos fases. En la primera, se realizó una revisión documental de antecedentes contextuales sobre el rol y responsabilidad jurídica actual ligada a las atribuciones de los municipios con relación al turismo, para posteriormente, desarrollar la segunda fase, la cual está orientada a describir una serie de indicadores, con el propósito de generar una caracterización de la forma que los municipios están gestionando el turismo a nivel local, generando una relación y reflexión a partir del trabajo etnográfico realizado por el investigador. Culminando con las principales conclusiones de lo anteriormente expuesto.

Finalmente, y con relación a los procedimientos éticos, de confiabilidad y validez en una investigación, está dada por tres criterios; credibilidad, transferibilidad y dependibilidad (Valles, 1999). teóricos e institucionales, los cuales dan cuenta sobre la realidad a investigar. 
- La credibilidad se relaciona con la experiencia del investigador que desarrollo el estudio, destacando el interés sobre esta temática en particular y trabajo realizado como profesional a cargo del turismo municipal en dos comunas de regiones distintas a nivel nacional, específicamente, en la Municipalidad de Petorca (2011-2012) y en la Municipalidad de los Vilos (2013)

- La transferibilidad según Valles (1999), depende de los procedimientos de muestreo, que en este caso se fundamentan con la estrategia metodológica utilizada y descrita anteriormente, orientada a la revisión de documentos teóricos e institucionales, los cuales dan cuenta sobre la realidad a investigar.

- La dependibildad estará dada a partir del trabajo en conjunto con otros investigadores del Centro de Investigación en Turismo y Patrimonio (CITYP) de la Región de Valparaíso, específicamente en la generación y participación de instancias de reflexión.

\section{PRINCIPALES RESULTADOS.}

Los principales resultados que dan cuenta y que permiten poder observar el rol que tienen los municipios en la gestión local del turismo, se estructura a partir de los objetivos específicos planteados. El primer tema abordado identifica las atribuciones legales y funciones que tienen los municipios para desarrollar el turismo. Posteriormente se identifican los diversos instrumentos de planificación a nivel local, resaltando la relevancia para estas instituciones y sus territorios contar con un "Plan de Desarrollo Turístico Comunal". Finalmente, se caracteriza a través de una serie de indicadores la gestión local turística de los municipios.

3.1 El Municipio: Un actor clave para la gestión local del turismo. El desarrollo del turismo en los diversos territorios tienen un actor relevante en los ámbitos vinculados a la gestión, planificación y desarrollo de esta actividad a nivel local, ya que conocen la oferta, atributos y potencialidades que tienen para su desarrollo desde una perspectiva territorial, nos referimos específicamente al "municipio". Según la actual constitución las municipalidades son definidas de acuerdo al inciso cuarto del artículo 118, como "corporaciones autónomas de derecho público, con personalidad jurídica y patrimonio propio, cuya finalidad es satisfacer las necesidades de la comunidad local y asegurar su participación en el progreso económico, social y cultural de la comuna" (Subsecretaría de Desarrollo Regional y Administrativo, 2010, p. 8). En el caso de nuestro pais, las municipalidades son unos de los órganos del Estado de carácter descentralizado y de más larga trayectoria en Chile. Esta situacion se puede apreciar en el origen de las comunas como unidad territorial y de las municipalidades como su órgano de gobierno, el cual data desde la época de la colonia, específicamente durante los años conocidos como la "Organización de la Republica", donde la 
constitución de 1833 creó la unidad territorial de los departamentos, regidos por municipalidades donde sus autoridades eran designadas. Este es un punto de inflexión, ya que tuvieron que pasar varias décadas para un cambio de carácter estructural a nivel local-comunal. Este proceso fue impulsado por la ley de organización de atribuciones de las municipalidades, más conocida como "La ley de la comuna autónoma", la cual además es reconocida como una normativa que permitió asentar las bases de la comuna como unidad básica de división territorial, gobernadas por municipalidades que gozan de cierto grado de autonomía con relación al gobierno nacional (Vial, 2014). En la actualidad las municipalidades están a cargo de la administración y gobierno de las 346 comunas existentes en el pais, incluyendo la Antártica de Chile, la cual está a cargo de la municipalidad de Cabo de Hornos, habiendo un total de 345 municipalidades que se agrupan en 54 provincias (Sinim, 2014). En los últimos 25 años se promulgaron leyes que generaron diversos impactos en el quehacer municipal. Un ejemplo que se puede observar es la ley 19.280 promulgada en el año 1993, la cual otorgo a las municipalidades mayor autonomía para fijar remuneraciones y modificar su personal. Otro ejemplo se puede visualizar en la ley 19.602 del año 1999, la cual generó una ampliación de sus facultades, reconociendo una serie de funciones privativas, vale decir, "exclusivas de los municipios", entre las cuales podemos mencionar; El desarrollo, aprobación y modificación del Plan de Desarrollo y Regulador Comunal (PLADECO), elaboración del presupuesto municipal, la generación de ordenanzas y disposiciones relacionadas con el manejo de la basura y acciones relacionadas con la planificación y regulación del espacio comunal y promover el desarrollo comunitario y local.

Adicionalmente se reconocieron una serie de funciones compartidas, vale decir, que "no son exclusivas del municipio", entre las cuales se destacan la promoción del empleo y del fomento productivo, capacitación y desarrollo del capital humano, desarrollo de viviendas sociales, seguridad ciudadana, salud, educación pre-escolar, básica y media, desarrollo económico y "Turismo". Esto último se observa específicamente en la legislación actual, la cual indica que "las municipalidades en el ámbito de su territorio, podrán desarrollar directamente o con otros órganos de la administración del Estado funciones relacionadas con el turismo, deporte y recreación" (Ley $N^{\circ} 18.695$, 2006, Letra e).

Es por este motivo que se puede visualizar que en muchas ocasiones, las acciones vinculadas principalmente a las funciones compartidas (no exclusivas), como es en este caso específico a las relacionadas con el desarrollo del turismo, el municipio actúa como ejecutor de las preferencias y/o disposiciones del gobierno central. Esta situacion se puede observar en las diversas instituciones y organizaciones que intervienen en los procesos de planificación y desarrollo de la actividad turística a nivel local, la que se debe materializar en todas las comunas con la participación de diversos actores. A continuación se presenta un cuadro con los diversos actores (públicos/privados) 
que participan en el proceso de planificación y desarrollo turístico, compuesto por instituciones públicas, unidades internas de los municipios y actores externos.

\section{Cuadro $\mathrm{N}^{\circ} 2$ : Actores que participan en el proceso de planificación turística a nivel local.}

\begin{tabular}{|c|c|c|}
\hline Instituciones Publicas & Unidades Gobierno Local & Actores externos \\
\hline Gobiemo Regional & Alcaldia & $\begin{array}{l}\text { Organizaciones de la sociedad civil } \\
\text { funcionales y territoriales (JJVV, Clubes, etc.) }\end{array}$ \\
\hline Gobierno Provincial & Concejo Municipal & $\begin{array}{l}\text { Organizaciones Gremiales del } \\
\text { sector turistico }\end{array}$ \\
\hline Municipios Vecinos & Administracióna Municipal & Empresas Privadas \\
\hline $\begin{array}{l}\text { Servicio Nacional de Turismo } \\
\text { (SERNATUR) }\end{array}$ & $\begin{array}{l}\text { Secretaria de Planificación Comunal (Secplac), } \\
\text { Unidad de Turismo y otras unidades del municipio }\end{array}$ & $\begin{array}{l}\text { Universidades e institutos } \\
\text { profesionales; Centros đe Investigación y Desarrollo. }\end{array}$ \\
\hline $\begin{array}{l}\text { Consejo Nacional de ta } \\
\text { Cultura y las Artes (CNCA) }\end{array}$ & $\begin{array}{l}\text { Dirección de Desarrollo Comunitario (Dideco); } \\
\text { Unidad de Turismo, Unidad de Cutura. }\end{array}$ & $\begin{array}{l}\text { Centros de Investigación y } \\
\text { Desarrollo y organizaciones culturales funcionales y } \\
\text { territoriales. }\end{array}$ \\
\hline $\begin{array}{l}\text { Instituto de Desarrollo } \\
\text { Agropecuario (INDAP) }\end{array}$ & $\begin{array}{l}\text { Dirección de Desarrollo Communtario (Dideco), } \\
\text { Programa Prodesal, Unidad de Turismo. }\end{array}$ & $\begin{array}{l}\text { Organizaciones no } \\
\text { Gubemamentales (ONG) }\end{array}$ \\
\hline SERCOTEC - CORFO & $\begin{array}{l}\text { Oficina de Promoción Productiva (Odepro), } \\
\text { Unidad de Turismo }\end{array}$ & $\begin{array}{l}\text { Fundaciones y organizaciones } \\
\text { empresariales micro y pymes }\end{array}$ \\
\hline $\begin{array}{l}\text { Ministerio de Obras Publicas } \\
\text { (MOP) - Ministerio de Medio Ambiente (MMA) }\end{array}$ & $\begin{array}{r}\text { Secretaria de Planificación Comunal (Secplac) y } \\
\text { otras unidades del municipio como la Unidad de Medio Ambiente }\end{array}$ & $\begin{array}{l}\text { Organizaciones no } \\
\text { Gubernamentales (ONG) }\end{array}$ \\
\hline $\begin{array}{l}\text { Instituto Nacional de } \\
\text { Estadisticas (INE) }\end{array}$ & $\begin{array}{l}\text { Secretaria de Planificación Comunal (Secplac) y } \\
\text { otras unidades đel municipio. }\end{array}$ & $\begin{array}{l}\text { Organizaciones no } \\
\text { Gubernamentales (ONG) }\end{array}$ \\
\hline Investigaciones-Carabineros & Unidad de Seguridad Ciudadana & Comités de Seguridad Ciudadana \\
\hline $\begin{array}{l}\text { Instituto Nacional de la } \\
\text { Juventud (INIUV) }\end{array}$ & $\begin{array}{l}\text { Dirección de Desarrollo Comunitario (Dideco-- } \\
\text { Cultura) }\end{array}$ & $\begin{array}{l}\text { Organizaciones de la sociedad civil } \\
\text { funcionales y territoriales Organizaciones no } \\
\text { Gubernamentales (ONG) }\end{array}$ \\
\hline $\begin{array}{l}\text { Ministerio de Modio } \\
\text { Ambiente MMA }\end{array}$ & $\begin{array}{c}\text { Secretaria de Planificación Comunal (Secplac) y } \\
\text { otras unidades del municipio como la Unidad de Medio Ambiente, } \\
\text { Transito. }\end{array}$ & $\begin{array}{l}\text { Organizaciones de la sociedad civil } \\
\text { funcionales y territoriales Organizaciones no } \\
\text { Gubernamentales (ONG) }\end{array}$ \\
\hline DIBAM & $\begin{array}{l}\text { Dirección de Desarrollo Comunitario (Dideco } \\
\text { Cultura y Educación) }\end{array}$ & $\begin{array}{l}\text { Organizaciones de la sociedad civil } \\
\text { funcionales y territoriales Organizaciones no } \\
\text { Gubemamentales (ONG) }\end{array}$ \\
\hline $\begin{array}{l}\text { MIDEPLAN-SENAMA- } \\
\text { EOSIS-INJUV }\end{array}$ & $\begin{array}{l}\text { Dirección de Desarrollo Comunitario (Dideco- } \\
\text { Cultura; Dirección de Aseo y Omato, entre otros) }\end{array}$ & $\begin{array}{l}\text { Organizaciones de la sociedad civil } \\
\text { funcionales y territoriales Organizaciones no } \\
\text { Gubernamentales (ONG) }\end{array}$ \\
\hline
\end{tabular}

Fuente: Elaboración propia a partir del trabajo desarrollado en el "Manual de Orientaciones para el Diseño de Planes de Desarrollo Turístico" (2015).

En el cuadro $\mathrm{N}^{\circ} 2$, se logra apreciar la complejidad que posee la actividad turística, visualizando la multiplicidad de actores que deben ser parte de cualquier estrategia de planificación y desarrollo turístico a nivel local, donde el papel de los municipios en este tipo de procesos es indispensable, considerando además la heterogeneidad de las capacidades y potencialidades que poseen las comunas a nivel nacional.

Asumiendo esta multiplicidad de actores, es pertinente referirse al Servicio Nacional de Turismo (SERNATUR), ya que es la institución pública encargada a nivel nacional 
de implementar las políticas públicas vinculadas al sector turístico, las cuales vienen direccionadas desde el nivel central del Gobierno de Chile para propiciar el desarrollo y crecimiento de la actividad turística. Un área de trabajo de esta institución, está focalizada en desarrollar y diversificar la oferta turística del país a través de normas y estándares de sustentabilidad y calidad, donde se aprecia al municipio como un actor clave, considerando su función en la planificación y gestión local del turismo, las atribuciones que posee y el tipo de decisiones que pueden tomar en áreas como; Infraestructura, señaletica, ordenanzas municipales, mejoramiento de áreas verdes, protección del patrimonio natural y cultural, entre otras. Este trabajo se ha potenciado con en el Programa de Turismo Municipal que SERNATUR viene implementando desde el año 2011, cuyo objetivo es;

"Lograr que los municipios incorporen la actividad turística dentro de su gestión anual como un tema relevante, comprendiendo que el fortalecimiento y desarrollo de esta actividad se logra cuando se hace en forma sistemática y asociativa, ya que toda acción que se emprenda a nivel local puede tener implicancia a nivel regional y nacional" (Sernatur, 2016).

En el cuadro $\mathrm{N}^{\circ} 2$, se logra apreciar la complejidad que posee la actividad turística, visualizando la multiplicidad de actores que deben ser parte de cualquier estrategia de planificación y desarrollo turístico a nivel local, donde el papel de los municipios en este tipo de procesos es indispensable, considerando además la heterogeneidad de las capacidades y potencialidades que poseen las comunas a nivel nacional.

Asumiendo esta multiplicidad de actores, es pertinente referirse al Servicio Nacional de Turismo (SERNATUR), ya que es la institución pública encargada a nivel nacional de implementar las políticas públicas vinculadas al sector turístico, las cuales vienen direccionadas desde el nivel central del Gobierno de Chile para propiciar el desarrollo y crecimiento de la actividad turística. Un área de trabajo de esta institución, está focalizada en desarrollar y diversificar la oferta turística del país a través de normas y estándares de sustentabilidad y calidad, donde se aprecia al municipio como un actor clave, considerando su función en la planificación y gestión local del turismo, las atribuciones que posee y el tipo de decisiones que pueden tomar en áreas como; Infraestructura, señaletica, ordenanzas municipales, mejoramiento de áreas verdes, protección del patrimonio natural y cultural, entre otras. Este trabajo se ha potenciado con en el Programa de Turismo Municipal que SERNATUR viene implementando desde el año 2011, cuyo objetivo es;

"Lograr que los municipios incorporen la actividad turística dentro de su gestión anual como un tema relevante, comprendiendo que el fortalecimiento y desarrollo de esta actividad se logra cuando se hace en forma sistemática y asociativa, ya que toda acción que se emprenda

a nivel local puede tener implicancia a nivel regional y nacional" (Sernatur, 2016). 


\section{Tabla Nº1: Objetivos Programa de Turismo Municipal.}

1. Lograr que los municipios consideren el turismo como una actividad relevante de la gestión municipal, tanto desde lo receptivo, como de lo emisivo.

2. Capacitar al capital humano de los municipios para que tengan la capacidad de intervenir y coordinarse técnicamente en materias de turismo.

3. Reforzar la implementación del Sistema de Calidad y Certificación de SERNATUR

4. Unificar imagen y promoción del destino en coherencia con la Política Nacional de Turismo

5. Estimular la asociatividad de micro y medianas empresas turísticas con el fin de generar productos turísticos para el turismo local, nacional e internacional.

6. Impulsar el desarrollo turístico sustentable en el territorio comunal

7. Generar información para la toma de decisiones en la actividad turística

8. Fomentar la Conciencia Turística en la comunidad local

Fuente: Elaboración propia a partir de la información extraída del sitio web institucional del Servicio Nacional de Turismo.

Con relación a las funciones que tienen los municipios para el desarrollo de la actividad turística a nivel local, se identificaron nueve áreas de acción para la gestión local del turismo a partir del trabajo desarrollado por Boullon (1990), adaptándolo a la realidad chilena y asumiendo que en el nivel operativo/práctico, y como se reflejó en la identificación de actores, el turismo es una actividad donde existe una co-responsabilidad con otras instituciones y organizaciones para su desarrollo.

\section{Cuadro No 3: Áreas de acción del municipio para el desarrollo del turismo.}

\begin{tabular}{|c|}
\hline ÁREAS DE ACCIÓN \\
\hline $\begin{array}{l}\text { Información. Obtener y recopilar información para elaborar un inventario turistico de la comuna que es } \\
\text { responsabilidad de la Unidad de Turismo y que debe transmitir esa información al organismo provincial, regional y a los } \\
\text { servicios que desarrollan la actividad turistica a nivel local. }\end{array}$ \\
\hline $\begin{array}{l}\text { Facilitación. Aplicar las normas y criterios sugeridos por los organismos nacional, regional y provincial } \\
\text { Sancionar y aplicar normas y criterios propios (que no se contradigan con los de los otros organismos) para resolver sin } \\
\text { demoras cualquier tipo de problemas que los turistas tengan durante su estadia. }\end{array}$ \\
\hline $\begin{array}{l}\text { Promoción. Definir la imagen turistica del municipio en relación con la demanda, con sus atractivos, su planta } \\
\text { turistica y su tipologia funcional / Seleccionar las plazas de mercado emisor al municipio en las que centrara su promoción / } \\
\text { Realizar eventos de comercialización en el ámbito del municipio / Organizar viajes de familiarización / Elaborar material } \\
\text { informativo para el turista real, consumidor potencial / Coordinar su acción con otros niveles (regional/nacional) y con la } \\
\text { actividad privada / Emprender, con otros municipios complementarios, acciones comunes de promoción, difusión y } \\
\text { ejecución del turismo social y familiar. }\end{array}$ \\
\hline $\begin{array}{l}\text { Fomento de Inversiones. Impulsar la radicación de capitales extranjeros y nacionales, en coordinación con el } \\
\text { organismo provincial - regional / Aplicar en su jurisdicción las estrategias nacionales y regionales / Adaptar sus acciones a } \\
\text { las politicas nacionales y regionales de desarrollo del espacio turístico / Realizar evaluaciones técnicas, económicas, y } \\
\text { financieras de los proyectos de inversión localizados en los municipios. }\end{array}$ \\
\hline $\begin{array}{l}\text { Contralor de Servicios. Aplicar las normas de control y sanciones elaboradas y aconsejadas por los organismos a } \\
\text { nivel nacional y regional / Mantener actualizado el inventario turistico mediante un registro municipal de servicios turisticos } \\
\text { Recibir, procesar y resolver las quejas por los turistas acerca del funcionamiento, precios y calidad de los servicios } \\
\text { turisticos locales. }\end{array}$ \\
\hline
\end{tabular}




Capacitación. Detectar las necesidades de capacitación del municipio e informar de las misma al organismo regional / Colaborar
en la puesta en marcha, en el ámbito del municipio, de los programas de capacitación elaborados por el organismo regional / Organizar
cuando el presupuesto lo permita programas de capacitación propios / Capacitar a su propio personal / Difundir en su ámbito la información
sobre becas y cursos de especialización nacionales y extranjeros.
Asistencia Téenica. Asociarse con otros municipios para afrontar problemas técnicos comunes / Solicitar asistencia técnica al
organismo regional de turismo o por su intermedio al organismo nacional. Usar otros Programas del nivel nacional, como el programa de
capacitación municipal de la Subsecretaria de Desarrollo Regional (SUBDERE)
Planificación e Investigación. Obtener datos cuantitativos sobre la demanda real, sobre el perfil y nivel de satisfacción del turista
Colaborar en la investigación sobre el grado de modernización y la rentabilidad de los servicios turísticos / Elaborar cl plan municipal de
desarrollo turistico / Asociarse con otros municipios para planificar la solución de problemas comunas / Asignar recursos para la puesta en
marcha de proyectos pilotos seleccionados por el plan / Definir con la actividad privada los problemas técnicos que afectan al sector y
encarar su solución por si mismo o a través de los organismos nacionales, regionales o internacionales / Realizar acuerdos para resolver
problemas cuya solución requiere la participación de otros organismos / Formular proyectos que puedan optar a linanciamientos externos de
carácter multiescalar. $A$ fondos de decisión regional (Fondo Nacional de Desarrollo Regional. FNDR; Fondo para la Innovación de la
Competitividad (FIC). A fondos de decisión nacional, INNOVA CORTO, FONDEF, CONICYT entre otros, A fondos de cooperación
internacional. (MERCOSUR. OCD, Francia, España entre otros)
Recreación. Promover la realización de actividades recreacionales para la población local, que puedan a la vez ser aprovechadas
por los turistas

Fuente: Elaboración propia a partir del trabajo desarrollado por Roberto C .Boullon (1990).

\subsection{Instrumentos de planificación municipal y su vinculación con la actividad turística.}

Los instrumentos de planificación municipal son fundamentales para generar procesos de "desarrollo local" en los diversos sectores socio-productivos presentes a nivel comunal, el cual se comprende como;

"Proceso reactivador de la economía y dinamizador de la sociedad local mediante el aprovechamiento eficiente de los recursos endógenos existentes en una determinada zona, capaz de estimular y diversificar su creciente economía, crear empleo y mejorar la calidad de vida de la comunidad local, siendo el resultado de un compromiso en el que se entiende como espacio de solidaridad activa" (Comité Económico y Social de las Comunidades Europeas, 1995; citado por: Zuñiga, 2013:11).

El turismo como actividad socio-económica creciente a nivel nacional, genera la necesidad de que los municipios tengan un protagonismo en los procesos de desarrollo a nivel local, a partir de las potencialidades y oportunidades que brinda el territorio comunal, 
siendo fundamentales los instrumentos de planificación existentes. Al observar la ley Nro.18.695 de "Orgánica Constitucional de Municipalidades", reconoce tres grandes instrumentos de planificación comunal con los que cuentan los municipios, estos son; el Plan de Desarrollo Comunal (PLADECO), el Plan Regulador Comunal y el Presupuesto Municipal.

De acuerdo a la actual legislación, el PLADECO es el instrumento rector que tienen los municipios, el cual a partir de su carácter indicativo, orienta el desarrollo comunal a través de la generación de estrategias y políticas, las cuales canalizan la gestión de la administración municipal, con el propósito de satisfacer las necesidades de comunidad local y promover su avance económico, social y cultural. Este instrumento de planificación comunal se desarrolla e integra a la comunidad y grupos focales, en seis áreas temáticas: Cultura y Vecindad; Turismo y habitabilidad; Patrimonio Inclusivo; Integración SocioEconómica; Gobernanza y Participación Ciudadana; Ciudad y Territorio. (Negrete, 2015).

En definitiva, se aprecia que a partir de su transversalidad y carácter estratégico que tiene este instrumento, el que se traduce en un documento que contiene la hoja de ruta y los fundamentos para la priorización de proyectos de inversión, convirtiéndose en la base para la conformación del presupuesto municipal anual de cada uno de los municipios del pais, el cual se presenta y debe ser aprobado por el concejo municipal.

Estos instrumentos se vinculan directamente con la capacidad de desarrollo de la actividad turística, ya que ponen en marcha diversas acciones impulsadas por la gestión municipal, entre las cuales podemos mencionar; La generación de ordenanzas municipales, gestión y regulación ambiental, ordenamiento territorial, generación de señaléticas, mejoramiento de infraestructura, fomento productivo, incentivos para el emprendimiento, educación y consciencia turística por parte de la población.

\subsection{PLADETUR: La herramienta estratégica para la planificación del turismo a nivel local.}

Con relación a la actividad turística, podemos apreciar la existencia del Plan de Desarrollo Turístico Comunal (PLADETUR). Este instrumento de planificación de carácter "indicativo", es clave para generar procesos de desarrollo turístico desde los territorios locales y desde un enfoque sustentable (Rivas, 2016), donde el municipio debería tener la capacidad de establecer los lineamientos estratégicos del desarrollo turístico en un territorio acotado, como lo es el "comunal", definiendo objetivos, políticas, estrategias y acciones, que orienten y guíen los ámbitos vinculados a la inversión socio-económica, al ordenamiento y uso de los recursos patrimoniales, culturales y naturales utilizables en la actividad turística, donde cada sistema/actor, ya sea comunidad, empresas turísticas e 
institucionalidad publica, tenga claridad de su rol como agente de desarrollo, facilitando por un lado, un mayor control de expectativas por parte de las comunidades, y por otro lado, poder generar las acciones orientadas de minimizar los impactos que se pudiesen generar en el entorno.

Otra característica del PLADETUR se vincula con la necesidad de articular una oferta turística de acuerdo a los potenciales de demanda, con el objetivo de generar un flujo constante y permanente de visitantes durante todo el año. De esta manera se generan nuevos empleos y se pone en valor de manera sustentable al patrimonio natural y cultural de las áreas urbanas y rurales de las comunas. (Negrete et al, 2015).

A partir del trabajo desarrollado por el SERNATUR, específicamente en el "Programa de Turismo Municipal", surgió la necesidad por parte de los coordinadores municipales de turismo (CMT) el tener información que sea útil y funcional para la generación de procesos de planificación y así poder facilitar la toma de decisiones para fortalecer la actividad turística a nivel comunal, desarrollando el manual "Orientaciones para el Diseño de un Plan de Desarrollo Turístico en Destinos Turísticos (PLADETUR)" editado el año 2015. Este trabajo identifica cinco etapas que son parte del proceso de elaboración de esta herramienta.

\section{Cuadro Nº 4: Etapas del proceso de elaboración y ejecución del (PLADETUR).}

\begin{tabular}{|c|}
\hline $\begin{array}{l}\text { Asociación de grupos locales. Etapa orientada en crear una estructura organizativa que por un lado supervise y coordine a los diversos } \\
\text { actores privados y públicos que forman parte del proceso turístico, con el objetivo de generar una "imagen -objetivo" compartida y validada sobre las expectativas } \\
\text { de desenvolvimientos de la actividad turistica por parte de los diversos actores. }\end{array}$ \\
\hline $\begin{array}{l}\text { Análisis de temas claves para la comunidad. Comienza una vez conformada la estructura organizativa, dando inicio a un } \\
\text { procesos de análisis con los actores locales vinculados con la actividad turistica, por cuanto se espera que las decisiones de los goblernos locales poseerán } \\
\text { mayor capacidad de éxito ya que podrán responder a las expectativas de la comunidad (residentes) y de los usuarios de la planta turistica a nivel local. }\end{array}$ \\
\hline $\begin{array}{l}\text { Planificación para la Acción. Está focalizada en formular el plan consensuado entre los diversos actores, teniendo que realizar tres } \\
\text { acciones concretas; 1) Definir objetivos especificos para el sector turismo, en función de la imagen -objetivo previamente establecida; 2) Generación de } \\
\text { metas y acciones con la determinación de plazos para su ejecución; y c) definición de estrategias y responsables de los diferentes actores a través de un } \\
\text { trabajo asocjativo. }\end{array}$ \\
\hline $\begin{array}{l}\text { Implementación y seguimiento. Por un lado la implementación está vinculado al diseño y definición de los procedimientos administrativos } \\
\text { por parte del gobierno local con el objetivo de facilitar y permitir una gestión asociativa con los actores locales, a partir del establecimiento de acuerdos para la } \\
\text { definición de responsabilidades para la realización y ejecución de proyectos y programas que corresponderá generar y finaneiar a cada una de las partes } \\
\text { involueradas en el plan, por otro lado está el seguimiento el cual implica la generación de métodos y procedimientos de registro sistemático de todas las } \\
\text { actividades realizadas y de los efectos que causan. de tal manera de tener información que permita poder reconducir acciones si es necesario. }\end{array}$ \\
\hline $\begin{array}{l}\text { Evaluación y retroalimentación. Fsta última ctapa permitirá poder obscrvar cl grado de cumplimiento de las metas y objetivos del } \\
\text { plan, facilitando cl análisis del impacto de cada una de las decisiones. Fsta última ctapa se considera relcvante y fundamental ya que con la información } \\
\text { generada se podrá generar camblos o reconducir la asignación de recursos, considerando que "el slstema de retroalimentación debe dar paso a una } \\
\text { planificación o acción subsiguiente" (ICLEJ, 1996: 11). }\end{array}$ \\
\hline
\end{tabular}

Fuente: Elaboración propia a partir de la información para el Diseño de un Plan de Desarrollo Turístico extraída del manual de

"Orientaciones (PLADETUR)". 
En definitiva, se observa este trabajo como una oportunidad concreta para el incremento de las capacidades y conocimiento por parte de los coordinadores municipales de turismo (CMT) a nivel nacional sobre esta herramienta. De esta manera se puede apreciar este documento como un insumo relevante para el desarrollo de este tipo de acción local, desarrollando además la capacidad de evaluar con anticipación las condiciones del territorio para desarrollar la actividad turística, lo que a su vez permitirá disponer de información pertinente y apropiada para seleccionar las iniciativas y proyectos más compatibles con las características del ecosistema a nivel local y así poder anticiparse a los impactos del ciclo de crecimiento de una zona turística (Butler,1980, Rivas 1994; OMT, 1998).

En este punto es relevante poder ahondar en la pertinencia que tiene el desarrollar un PLADETUR porlos municipiosen Chile, yaqueapesarqueno sereconozca esteinstrumento en la actual legislación de municipalidades, es la principal herramienta para el desarrollo estratégico a nivel comunal de la actividad turística. Por lo tanto, el mayor o menor impacto que pueda generar la elaboración de dicha herramienta, dependerá de la capacidad de instalar sus resultados, tanto en el Plan de Desarrollo Comunal (PLADECO) que está en la base del presupuesto municipal anual; en el Plan Regulador Urbano Comunal y en función de su imagen objetivo que define la normativa que determina el ordenamiento del territorio y sus respectivos usos de suelo, y en las ordenanzas municipales. (Negrete et al, 2015).

Otro aspecto que debe abordar un PLADETUR, se vincula con la elaboración de un sistema de gobernanza que permita articular o coordinar un desarrollo integral del territorio urbano y rural, a fin de establecer salvaguardias eficaces del patrimonio natural y cultural tangible e intangible (modo de vida) y encauzar, normar, monitorear y controlar el uso del territorio por otras actividades (Negrete et al, 2015), siendo un aspecto clave para la sustentabilidad de la actividad.

Siguiendo esta misma línea, es importante destacar que el como instrumento de planificación y ordenamiento del territorio para la actividad turística PLADETUR se puede elaborar en la actualidad considerando más de una comuna. Esta situacion se puede apreciar en Chile donde en casos específicos ha tomado el rol como instrumento "intercomunal", atravesando una comuna específica, generando estrategias, objetivos, acciones y productos turísticos que tienen la capacidad de integrar un conjunto de territorios comunales. Un ejemplo concreto es lo que aconteció durante el año (2015), donde se dio inicio al "Plan de Desarrollo Intercomunales de Turismo Sustentable de Chiloé". Estos planes tienen como objetivos diseñar un plan estratégico para la imagen del territorio, el diseño de productos turísticos y culturales, realizar y promover estrategias y programas de educación y recreación, de fomento productivo, de temas medioambientales y de desarrollo local con un enfoque sustentable. Esto se ha traducido en la práctica en el alineamiento de forma conjunta entre los municipios de Dalcahue, 
Ancud y Quemchi, específicamente en la distribución de responsabilidades y funciones que permitan realizar una gestión más eficiente y que se traduzcan en economizar costos, racionalización de los recursos, agilizar procesos de desarrollo vinculado al sector turístico, generando un impacto en el desarrollo socioeconómico a partir de esta integración de las comunas en el ámbito geográfico y político- administrativo.

Adicionalmente, la elaboración de esta herramienta de planificación turística tiene la misión de vincularse como un marco de referencia para la elaboración y ejecución de sus acciones y objetivos con los instrumentos de planificación de carácter regional y nacional, tales como las Estrategias Regionales de Desarrollo, las Estrategias Regionales de Innovación para la Competitividad, los Planes de Acción para el desarrollo turístico de nivel regional de SERNATUR, los instrumentos de planificación de la Subsecretaria de Turismo para el desarrollo de zonas de intereses turístico (ZOIT) y las Estrategias Nacionales de Turismo.

Finalmente, cada uno de estos instrumentos deben de tener la capacidad de dialogar y trabajar en conjunto, ya que son fundamentales para desarrollar de una manera integral la actividad turística, sirviendo como una especie de "guía", para que desde el municipio se puedan vincular acciones, optimizar recursos y focalizar la generación de nuevos destinos, productos y servicios turísticos en base a estudios que arrojen como resultados mercados emergentes, tendencias, perfiles de la demanda, estacionalidad, llegadas, pernoctaciones, oportunidades de inversión en infraestructura habilitante y nuevos requerimientos por parte de los turistas nacionales e internacionales.

\subsection{Caracterización de la gestión local del turismo en los municipios en Chile.}

Con el propósito de poder caracterizar la gestión local en turismo por parte de los municipios en Chile, se seleccionaron una serie de indicadores, los cuales son elaborados por el Servicio Nacional de Turismo (SERNATUR) en el marco de los informes anuales que realiza la institución para dar cuenta de la gestión de su programa de gestión municipal.

\section{Tabla N²: Indicadores de gestión municipal para el desarrollo turístico.}

\begin{tabular}{|l|}
\hline 1. Tipo de orgánica y/o unidad encargada del ámbito turistico. \\
\hline 2. Presupuesto asignado para la generación de acciones (no se considera el gasto en \\
honorarios). \\
\hline 3. Existencia de un coordinador municipal de turismo. \\
\hline 4. Profesión de los Coordinadores (CMT). \\
\hline 5. Rotación de los Coordinadores (CTM). \\
\hline 6. Cantidad de personas que trabajan en la instancia de turismo incluyendo al encargado. \\
\hline 7. Instrumentos de planificación utilizados preferentemente como referencia. \\
\hline 8. Municipios que cuentan con Plan de Desarrollo Turistico (Pladetur) \\
\hline
\end{tabular}

Fuente: Elaboración propia a partir de la información disponible por el Servicio Nacional de Turismo. 
A continuación se muestran los datos referentes a cada una de estos indicadores, siendo de gran utilidad y pertinencia analizarlos, ya que permiten dar respuesta al tercer objetivo específico propuesto, el cual está vinculado a caracterizar la labor que están cumpliendo los municipios en la gestión turística local. - Tipo de orgánica y/o unidad encargada del ámbito turístico. Con relación al tipo de orgánica y/o unidad de turismo podemos visualizar que la gran mayoría de los municipios tiene como unidad una oficina de turismo (154), lo que representa al 44, 63\% del total de municipios existentes en Chile. Posteriormente encontramos por un lado a los municipios que tienen oficinas de información turísticas (57) las cuales funcionan durante todo el año, y por otro lado a las oficinas de información turística que están operativas sólo en temporada alta (56). En un número menor se encuentran los municipios que poseen departamentos de turismo (21) y dirección de turismo (18). Es importante señalar que este grupo lo componen básicamente municipios que tienen una clara vocación turística, como por ejemplo Iquique, La Serna, Pichilemu, Villarrica, Viña del Mar, Panguipuli, Valparaíso, entre otros, , los cuales representan el 11,30\%. Finalmente se puede observar a (92) municipios que no cuentan con ningún tipo de unidad y que solo poseen un coordinador turístico (CMT).

Tabla $N^{\circ}$ 3: Tipo de unidad encargada del turismo.

\begin{tabular}{|c|c|}
\hline Tipo de Unidad encargada del Turismo & N \\
\hline Departamento de Turismo & 21 \\
\hline Dirección de Turismo & 18 \\
\hline Oficina de Turismo & 154 \\
\hline Oficina de Información Turistica & 57 \\
\hline Oficina de información turistica por temporada & 56 \\
\hline Municipios sin oficinas y que solo poseen CMT & 92 \\
\hline
\end{tabular}

Fuente: Elaboración propia a partir de los datos de Sernatur.

\section{- $\quad$ Presupuesto asignado para la generación de acciones.}

Si visualizamos un aspecto relevante y que incide directamente en la capacidad de gestión y acción que tienen los municipios, se vincula con la cantidad de recursos económicos otorgado para la generación de acciones en el área. Según los datos, el $40 \%$ tiene un presupuesto anual inferior a $\$ 1.000 .000$, mientras que el $23 \%$ cuenta con un presupuesto de $\$ 1.000 .0000$ a $\$ 5.000 .0000$, el $14,6 \%$ entre $\$ 5.000 .000$ a $\$ 10.000 .000$ y el $15,0 \%$ entre $\$ 10.000 .000$ a $\$ 30.000 .000$. Finalmente encontramos a los municipios que invierten más de $\$ 30.000 .000$ representado en el $7,5 \%$. 
Tabla N ${ }^{\circ}$ 4: Presupuesto directo asignado al durante el año 2015.

\begin{tabular}{|c|c|}
\hline Rangos de inversión & Porcentaje \\
\hline Menos de 1 Millos de Pesos & $39,90 \%$ \\
\hline Entre 1.000.001 y 5.000 .000 & $23,00 \%$ \\
\hline Entre 5.000.001 y 10.000 .000 & $14,60 \%$ \\
\hline Entre 10.000.001 y 30.000 .000 & $15,00 \%$ \\
\hline Más de 30.000.000 & $7,50 \%$ \\
\hline
\end{tabular}

Fuente: Elaboración propia a partir de los datos de Sernatur.

\section{- Coordinador Municipal de Turismo (CMT).}

Otro indicador vinculado a los recursos humanos de los municipios, se asocia a la presencia de un Coordinador Municipal de Turismo (CMT). Este funcionario es clave para desarrollar el turismo, el cual facilita los procesos de participación en instancias de desarrollo y de planificación turística a nivel local. A partir de los datos, se observa en el cuadro ${ }^{\circ} 1$ una cantidad importante de municipios a nivel nacional, específicamente (301 comunas) que representan el 87\%, que cuenta con un (CMT), mientras que (44 comunas) que representan el 13\% del total de municipios no cuenta con un (CMT). A pesar de la alta representatividad de este dato, se puede apreciar a (14 comunas) que nunca han nombrado a un coordinador municipal de turismo, donde se podría asumir que para temas vinculados con la actividad turística, eventualmente lo asume otro funcionario que no es especialista en el área.

Tabla N5: Coordinadores Municipales de Turismo (CMT).

\begin{tabular}{|c|c|c|}
\hline Presencia de (CMT) & N & Porcentaje \\
\hline Con coordinador municipal de turismo & 303 & $87 \%$ \\
\hline Sin coordinador municipal de Turismo & 42 & $13 \%$ \\
\hline Total & $\mathbf{3 4 5}$ & $\mathbf{1 0 0 \%}$ \\
\hline
\end{tabular}

Fuente: Sernatur.

\section{- $\quad$ Profesión de los Coordinadores Municipales de Turismo.}

Otro indicador vinculado a los recursos humanos se vincula con la pertinencia con relación a la profesión del funcionario municipal encargado del área. Se observa en la tabla $\mathrm{N}^{\circ} 5$ del cuadro $\mathrm{n}^{\circ} 1$ la presencia de (202 comunas) que representan el $66,6 \%$ del total municipios con (CMT) que no poseen una profesión vinculada al turismo, mientras que (101 comunas) que representan el 33,4\% los (CMT) poseen una profesión vinculada al turismo. Este dato se aprecia como una limitante, considerando la relevancia que tiene poseer conocimientos técnicos que permitan el 
buen desempeño y así poder generar procesos de desarrollo turístico a nivel comunal.

Tabla Nº: Profesión de los Coordinadores (CMT).

\begin{tabular}{|c|c|c|}
\hline Profesión del (CMT) & N & Porcentaje \\
\hline (CMT) con profesión vinculada al turismo & 101 & $33,40 \%$ \\
\hline (CMT) con profesión NO vinculada al turismo & 202 & $66,60 \%$ \\
\hline Total municipios con (CMT) & $\mathbf{3 0 3}$ & $\mathbf{1 0 0} \%$ \\
\hline
\end{tabular}

Fuente: Sernatur.

\section{- $\quad$ Rotación de los Coordinadores Municipales de Turismo (CTM).}

Con relación a la rotación o tiempo de permanencia, se visualiza que el $68,1 \%$ del total de los municipios que cuentan con un (CMT) tienen un tiempo de permanencia en el cargo que no supera los dos años, mientras que el 9,4\% tiene un tiempo de permanecía entre 3 a 4 años y el 10, 3\% de 5 a 6 años. Finalmente, podemos visualizar que solo el $12,2 \%$ del total de los municipios que cuentan con un tiempo de permanencia superior a 7 años en el cargo. Este es un dato relevante, considerando los impactos que tiene este indicador en la continuidad de los procesos de planificación estratégica en turismo.

Tabla Nº: Rotación de los Coordinadores (CMT).

\begin{tabular}{|c|c|}
\hline Rango de tiempo de permanencia en el cargo & Porcentaje \\
\hline Menos de 1 año & $31,00 \%$ \\
\hline Entre 1 a 2 años & $37,10 \%$ \\
\hline Entre 3 a 4 años & $9,40 \%$ \\
\hline Entre 5 y 6 años & $10,30 \%$ \\
\hline Entre 7 y 8 años & $2,80 \%$ \\
\hline Entre 9 y 10 años & $2,80 \%$ \\
\hline Más de 10 años & $6,60 \%$ \\
\hline
\end{tabular}

Fuente: Elaboración propia a partir de los datos de Sernatur.

\section{- Cantidad de personas que trabajan en la instancia de turismo incluyendo al encargado.}

Otro indicador vinculado con el recurso humano dispuesto por parte de los municipios, el cual identifica a las personas que trabajan en instancia de turismo incluyendo al (CMT), se aprecia que casi la mitad de los municipios en Chile, representado en un 48,4\%, solo cuenta con una persona responsable de esta área, mientras que en un poco más de un tercio, específicamente el 36,6\%, se encuentran trabajando 2 a 3 personas. En un 
porcentaje mucho menor están los municipios que tienen una cantidad importante de personas que trabaja en el área del turismo, donde el 10,3\% tienen entre 4 a 10 personas y el 1,9\% tiene más de 10 personas trabajando. Si se observa con más especificidad los datos, se pueden visualizar que los municipios que tienen sobre cuatro personas, son los que poseen una clara vocación turística, entre los cuales se encuentran; Valparaíso (15 personas), Puerto Natales (9 personas), Viña del Mar, Arica, Panguipulli, Ancud y Puerto Monnt (8 personas), Valdivia, providencia (7), Coquimbo, La Serena, Puerto Varas (6).

Tabla Nº ${ }^{\circ}$ Cantidad de personas que trabajan en la instancia de turismo incluyendo al encargado.

\begin{tabular}{|c|c|}
\hline $\begin{array}{c}\text { Rango de personas que trabajan en } \\
\text { Turismo }\end{array}$ & Porcentaje \\
\hline Ninguna & $2,80 \%$ \\
\hline 1 persona & $48,40 \%$ \\
\hline Entre 2 a 3 personas & $36,60 \%$ \\
\hline Entre 4 y 10 personas & $10,30 \%$ \\
\hline Más de 10 personas & $1,90 \%$ \\
\hline
\end{tabular}

Fuente: Elaboración propia a partir de los datos de Sernatur.

\section{- Instrumentos de planificación utilizados preferentemente como referencia.}

Si se observan los instrumentos de planificación utilizados por los municipios como referencias para el desarrollo de la actividad turística, se puede apreciar la carencia existente en la utilización del Pladetur con solo el 23,5\%, la política Regional de Turismo con el 21,6\% y la Estrategia Regional de Turismo con solo el 24,4\%, siendo el Plan de Desarrollo Comunal (PLADECO) con un 34,3\% el instrumento más utilizado como referencia por los municipios. Sin embargo, un dato que es preocupante, tiene relación a que casi un cuarto del total de los municipios específicamente el $24,9 \%$ no ha utilizado ningún tipo de instrumento de planificación para el área. 
Tabla Nº9: Instrumentos de planificación utilizados preferentemente como referencia.

\begin{tabular}{|c|c|}
\hline $\begin{array}{c}\text { Instrumentos de planificación utilizados preferentemente como } \\
\text { referencia }\end{array}$ & Porcentaje \\
\hline Política Regional de Turismo & $21,60 \%$ \\
\hline Estrategia Regional de Turismo & $24,40 \%$ \\
\hline Pladeco & $34,30 \%$ \\
\hline Pladetur & $23,50 \%$ \\
\hline Plan de Desarrollo & $8,50 \%$ \\
\hline Manual de Turismo Municipal & $0,50 \%$ \\
\hline Catastros & $1,90 \%$ \\
\hline Manual & $0,50 \%$ \\
\hline Otro (s) & $0,50 \%$ \\
\hline Total & $24,90 \%$ \\
\hline & $\mathbf{1 0 0} \%$ \\
\hline
\end{tabular}

Fuente: Sernatur.

\section{- Municipios que cuentan con Plan de Desarrollo Turístico (PLADETUR).}

Si observamos los datos del informe anual del programa de turismo municipal elaborado por el Servicio Nacional de Turismo (2015), podemos apreciar un déficit importante de planificación de carácter estratégico a nivel local en turismo por parte de los municipios en Chile, donde el 60,9\% (210 municipios) no cuentan con esta herramienta de planificación, mientras que el 16,5\% (57 municipios) se encuentra en proceso de elaboración y donde solo el 22,6\% (78 municipios) cuenta con en la actualidad con un PLADETUR.

Tabla $N^{\circ} 10$ : Municipios que cuentan con (Pladetur) a nivel nacional.

\begin{tabular}{|c|c|c|}
\hline Planes de Desarrollo Turístico & N & Porcentaje \\
\hline Municipios que cuentan con PLADETUR & 78 & $22,60 \%$ \\
\hline Municipios que están en etapa de elaboración & 57 & $16,50 \%$ \\
\hline Municipios que no cuentan con PLADETUR & 210 & $60,90 \%$ \\
\hline Total & $\mathbf{3 4 5}$ & $\mathbf{1 0 0} \%$ \\
\hline
\end{tabular}

Fuente: Elaboración propia a partir de los datos de Sernatur 


\section{CONCLUSIONES}

A partir de los datos e información descrita y analizada, se pudieron visualizar las diversas funciones que los municipios deben ejecutar para poder fomentar y desarrollar la actividad turística a escala local. Sin embargo, si uno revisa los datos, se puede observar la inconsistencia, entre el discurso que existe sobre la relevancia de la actividad turística para el desarrollo local de las comunas, y la forma en que los municipios están abordando el trabajo de este sector. Esta situacion se observa ya que no existe una real intención o priorización para desarrollar la actividad turística, asumiendo que los diversos municipios existentes a nivel nacional poseen características particulares que influyen en el desarrollo de este interés, las cuales se vinculan con sus ingresos, cantidad de habitantes, índice de pobreza y a la capacidad de atracción turística, tanto de visitantes, como de inversión pública/privada en comunas que presentan déficit de atractivos y/o recursos turísticos. Cada uno de estos factores impacta en las capacidades diversas que existen a nivel nacional para desarrollar el turismo a nivel comunal, considerando las contingencias a las que deben dar respuestas y la escasez de recursos asignados por el nivel central.

Se pudo constatar que el desarrollo de la actividad turística en el ámbito local es una labor compartida y "no exclusiva" de los municipios, donde participan una cantidad importante de actores que se vinculan con el desarrollo turístico. Es por este motivo que cobra mucha relevancia la capacidad de coordinación que puedan generar en distintos niveles, tanto internos vinculados a la "gestión municipal", como externos asociados a la relación que se debe generar con otras instituciones de carácter público y privado, y así poder alinear las múltiples acciones que se pueden producir, siendo fundamental potenciar su articulación, y así poder fomentar estrategias que integran la oferta turística basada en los elementos naturales, culturales y sociales que distinguen a cada territorio.

A partir de los datos observados sobre el déficit por parte de los municipios en generar procesos de planificación turística, considerando la importancia que tiene la elaboración y validación de los instrumentos de planificación descritos, siendo un desafío para el futuro el reconocimiento en la actual legislación de municipalidades el generar un (Pladetur), como también el poder establecer procesos de evaluación $\mathrm{y}$ seguimiento constante, siendo fundamentales e insustituibles poder reconocer la utilidad de estas herramientas para el desarrollo de la actividad turística.

Se asume en la actualidad la relevancia que tienen los municipios para el desarrollo de la actividad turística a nivel local, sin embargo, y como se pudo constatar en el transcurso de este artículo, se observa la necesidad de realizar transformaciones en las atribuciones jurídicas, vinculadas a posesionar esta actividad en la gestión municipal, con el propósito de mejorar tanto en aspectos de gestión interna, 
especialmente lo vinculado con la capacidad presupuestaria y de recursos humanos, como también en los procesos de planificación estratégica a nivel local del turismo, siendo en la actualidad estos aspectos una barrera para el desarrollo de la actividad.

Finalmente es importante reconocer que ninguna comuna está aislada en el territorio, por lo tanto, la labor que puedan cumplir las municipalidades van a producir impactos directos e indirectos en comunas que se encuentren cercanas o aledañas, ya que el turismo no tiene límites políticos-administrativos, entendiendo que un destino o producto turístico puede estar inserto en más de una comuna, en que la visita a atractivos y el uso de servicios generalmente están localizados en una ovarias comunas, generandoademás unanecesidad de comenzar a intensificar el trabajo inter-comunal para el desarrollo de la actividad en el futuro.

\section{BIBLIOGRAFÍA}

Biblioteca del Congreso Nacional de Chile. (s/f-f). Ley 19.452 de 16-04-1996: Modifica Disposiciones que indica la Ley No 18.695 , Orgánica Constitucional de Municipalidades. (Revisado el 30 de septiembre de 2016). Retrieved from http://www. leychile.cl/Navegar/?idNorma=30819\&idVersion=1996-04-16\&idParte Biblioteca del Congreso Nacional de Chile. (s/f-g). Ley 19.602 de 25-03-1999: Modifica la Ley No 18.695, Orgánica Constitucional de Municipalidades, en materia de Gestión Municipal. (Revisado el 30 de septiembre de 2016). Retrieved from http://www.leychile.cl/Navegar /?idNorma=134046\&idVersion=1999-03-25\&idParte

Boullon, R. (1990). Los Municipios Turísticos. Editorial Trillas, México.

Butler, R. (1980). The Concept of a Tourist Area Cycle of Evolution: Implications for Managements Resources. The Canadian Geographer. Vol. XXIV, 1, pp- 5 - 12, Canadá.

Canales J.M. y P. Pérez (2002). Introducción al Gobierno y a la Gestión Local, Editorial Club Universitario, Alicante.

Federación de Empresas de Turismo de Chile (FEDETUR - 2016). Barómetro Chileno del Turismo $\mathrm{N}^{\circ}$ 20, Marzo (2016).

Ley de Turismo, 2010, Art.2.

Márquez Fernández, D. (2002) (coord.). Nuevos horizontes en el desarrollo rural. Madrid: Akal.

Negrete, Jorge. (2015). Presentación en Simposio Turismo y Habitabilidad como Encargado CITYP del Eje Temático Turismo y Habitabilidad, PLADECO de Valparaíso 2016 -2023. Valparaíso, 2 de octubre 2015.

Negrete et al. (2015). "Diagnóstico y Plan de Desarrollo Turístico de Cartagena", 2016 - 2020, PUCV - CITYP, Chile.

Organización Mundial del Turismo (1998). Guide for Local Authorities on Developing Sustainable Tourism. Madrid, España. 1998.

Rivas, Humberto. (2016). Presentación Acto Inaugural del año académico de la Carrera 
de Turismo de la Universidad Andres Bello. Santiago, Chile.

Rivas, H (1994). Hacia un Desarrollo Turístico de los Ambientes Naturales en Chile. En: Ambiente y Desarrollo. Vol X. No 4. pp. 39 - 46. Santiago, Chile.

Sernatur (2015). Informe anual del programa de turismo municipal.

Sernatur (2014). Informe anual del programa de turismo municipal.

Sernatur \& Rivas (2015). Orientaciones para el Diseño de un Plan de Desarrollo Turístico en Destinos Turísticos (Pladetur).

Valles, M (1999). Técnicas Cualitativas de Investigación Social, reflexión metodológica y práctica profesional. Madrid, España: Ed.Sintesis.

Vial, C (2014). "Las Municipalidades y su papel en el contexto institucional en Chile". trabajo preparado para presentación en la mesa "diálogo sobre la descentralización municipal en argentina y chile", en el xi congreso chileno de ciencia política, organizado por la asociación chilena de ciencia política (accp). santiago, 15 al 17 de octubre de 2014.

Zuñiga González, C. A. (2013). Impacto de los Sistemas de Producción Agropecuarios en el Desarrollo Local Sostenible de Nicaragua, 1998- 2005: Índice de Malmquist DEA con un producto Orientado. Published in: Universitas (León), Vol. 1, No. 4 (10. November 2013):10-17. 